\title{
Le phénomène de « haine de soi juive »: de la douleur d'être Juif en Allemagne (1867-1933)
}

Das Phänomen des Jüdischen Selbsthasses - vom Leiden der deutschen Juden 1867-1933

'Jewish Self-Hatred' or the Pain of Being Jewish in Germany (1867-1933)

Martine Benoît

\section{CpenEdition}

\section{Journals}

Édition électronique

URL : https://journals.openedition.org/ceg/7476

DOI : $10.4000 /$ ceg.7476

ISSN : 2605-8359

Éditeur

Presses Universitaires de Provence

Édition imprimée

Date de publication : 25 septembre 2019

Pagination : 149-158

ISBN : 979-10-320-0234-6

ISSN : 0751-4239

Référence électronique

Martine Benoît, « Le phénomène de « haine de soi juive » : de la douleur d'être Juif en Allemagne (1867-1933) », Cahiers d'Études Germaniques [En ligne], 77 | 2019, mis en ligne le 25 mars 2021 consulté le 15 juin 2021. URL : http://journals.openedition.org/ceg/7476 ; DOI : https://doi.org/ $10.4000 /$ ceg.7476 


\title{
Le phénomène de « haine de soi juive »: de la douleur d'être Juif en Allemagne (1867-1933)
}

\author{
Martine BENOIT \\ Université de Lille / CECILLE (EA 4074)
}

Les critiques s'entendent généralement pour attribuer à Theodor Lessing la paternité de l'expression "Jüdischer Selbsthass », " haine de soi juive ». Né en 1872 à Hanovre au sein d'une famille juive acculturée, Theodor Lessing était un publiciste, essayiste et professeur de philosophie, démocrate convaincu, qui se battit dès le tournant du siècle pour les droits des femmes et l'éducation des couches défavorisées. Républicain engagé, il défendit avec un courage tant moral que physique une République de Weimar malade de ses faiblesses et de ses hésitations. Juif, il combattit l'antisémitisme en Allemagne et plaida pour un sionisme socialiste pour venir en aide aux Ostjuden. Il fut assassiné par des hommes de main des nazis le 31 août 1933 à Marienbad où il se pensait pourtant en sécurité. L'ouvrage Der jüdische Selbsthass paraît à la fin de l'année 1930, alors que l'Allemagne voit croître le nombre de députés nazis au Reichstag et avec lui, l'antisémitisme se diffuser toujours plus profondément dans la société. Dans son livre, pas très bien construit, qui aborde de nombreux sujets mais dont le propos central est d'encourager les Juifs allemands à développer leur estime de soi et à assumer pleinement leur judéité, Theodor Lessing présente tout d'abord la « haine de soi » comme un phénomène universel avant de se demander : "Comment est-il possible que tous les hommes s'aiment eux-mêmes mais que le Juif soit le seul à s'aimer si mal ${ }^{1}$ ? ». Mon propos n'est pas de présenter ici le livre de Theodor Lessing ${ }^{2}$ : je propose de réfléchir au phénomène de " haine de soi » dans la minorité juive allemande entre le début du Reich bismarckien et la fin de la République de Weimar en me servant de Theodor Lessing comme d'un fil conducteur.

Je voudrais faire encore deux remarques préliminaires : le terme, dont Bernard Banoun soulignait avec justesse l'impossible traduction ${ }^{3}$, porte une charge très

1. Theodor Lessing, Der jüdische Selbsthass, München, Matthes \& Seitz 1984 [1930], p. 47 (« Wie kommt es, dass alle Menschen sich selber lieben und nur der Jude liebt sich selber so schlecht? ») : on soulignera ici l'opposition malheureuse de l'homme et du Juif.

2. Voir Martine Benoit, "Theodor Lessing et le concept de "haine de soi juive" », in Esther Benbassa, Jean-Claude Attias, La haine de soi - Difficiles identités, Bruxelles, Complexe, 2000, p. 27-46.

3. Bernard Banoun, "À défaut d'une définition. Approches du Jüdischer Selbsthass », in Daniel Baric, Tristan Coignard, Gaëlle Vassogne (dir.), Identités juives en Europe centrale - des Lumières à 
forte en empruntant au substantif allemand de "Judenhass», nous verrons qu'il a été utilisé par certains comme synonyme d'« antisémitisme juif »; la « haine de soi » n'est pas uniquement juive ou judéo-allemande : le volume consacré par Esther Benbassa et Jean-Claude Attias à La haine de soi proposait d'ailleurs une «'déjudaïsation' de la problématique de la'haine de soi'4 » et abordait notamment l'anorexie féminine et l'homophobie gay ; déjà en 1947, Kurt Lewin évoquait le phénomène de « haine de soi » chez les noirs américains et les migrants italiens, polonais ou grecs de la deuxième génération ${ }^{5}$.

Je propose d'avancer pas à pas et de voir tout d'abord comment ce phénomène a été approché par les sciences humaines et sociales, comment cette haine de soi se serait exprimée dans la minorité judéo-allemande et enfin je ferai un focus sur les écrivains judéo-allemands et leur traitement littéraire de ce phénomène. Cet article s'interroge sur la justesse de l'expression du « jüdischer Selbsthass » pour savoir si elle est encore opérante, proposant d'emblée un sous-titre pour exprimer d'abord la « douleur » d'être Juif en Allemagne entre 1867 et 1933.

\section{Analyses du phénomène}

On peut partir d'une appréhension simple du phénomène de " haine de soi juive » en citant l'avant-propos qu'écrivait Theodor Lessing à son livre Der jüdische Selbsthass :

Auch der Verfasser hat in seiner Jugend eine Zeitspanne ausschließlicher Hingabe an das "Deutschtum", ausschließlicher Abwehr gegen das "Judentum" durchlaufen".

De nombreux chercheurs utilisent des expressions qui leur permettent d'approcher le phénomène ; je citerai les exemples les plus éclairants : I'historien Steven M. Lowenstein utilise le substantif de « Selbstverneiner » et développe son propos en parlant de « jüdische Ablehnung von Juden », $d^{\prime}$ « extreme Leugnung des Judentums » ou d' $\mathrm{d}^{\prime}$ Abwendung vom Judentum ${ }^{7}$ ", soulignant ainsi le rejet, ledésaveu,ledétachement;|'historienPeterGayévoqueun «antijüdischesGefühl ${ }^{8}$ », le Juif cherchant à fuir sa judéité, à renier son identité ${ }^{2}$; le psychologue américain

I'entre-deux-guerres, Tours, Presses Universitaires François-Rabelais, 2014, p. 179-202.

4. Jean-Claude Attias, "Conversion et haine de soi », in Benbassa, Attias, La haine de soi, p. 293-306 (ici p. 293).

5. Kurt Lewin, Die Lösung sozialer Konflikte - Ausgewählte Abhandlungen über Gruppendynamik, Bad Nauheim, Christian Verlag, 1953 (première édition en américain en 1948), notamment p. 262.

6. Lessing, Der jüdische Selbsthass, p. 40.

7. Steven M. Lowenstein, «Ideologie und Identität », in Steven M. Lowenstein, Paul Mendes-Flohr, Peter Pulzer, Monika Richarz (dir.), Deutsch-jüdische Geschichte in der Neuzeit, vol. 3, Umstrittene Integration 1871-1918, München, Beck, 1997, p. 278-301 (ces expressions se trouvent aux pages 284, 286 et 287).

8. Peter Gay, « Begegnung mit der Moderne - Deutsche Juden in der deutschen Kultur », in Werner E. Mosse, Arnold Paucker (dir.), Juden im wilhelminischen Deutschland 1890-1914, Tübingen, Mohr Siebeck, 1998, p. 241-311, ici p. 305.

9. Peter Gay, Freud, Juden und andere Deutsche - Herren und Opfer in der modernen Kultur, München, DTV, 1989 (la première édition en américain date de 1978), p. 212 (« seinem Jüdischsein 
Kurt Lewin parlait quant à lui de "Verachtung unter Juden ${ }^{10}$ », I'historien Jacob Toury de "Renegaten " ". Hans Dieter Hellige allait même jusqu'à évoquer un antisémitisme juif, un « innerjüdischer Antisemitismus ${ }^{12}$ ».

Les historiens s'entendent pour dire que le phénomène s'est étendu avec la grande dépression économique des années 1873-1896 et qu'il s'explique par des facteurs historiques, socio-économiques et psychologiques. L'historien Walter Grab souligne notamment la conjonction de deux moments : d'une part l'émancipation parcellaire des années 1860, où le judaïsme ne devient pas une religion égale aux deux confessions chrétiennes ; d'autre part la montée d'un antisémitisme racial et raciste qui s'organise peu à peu en mouvement politique ${ }^{13}$.

À ces facteurs historiques, Hans Dieter Hellige joint des causes plus psychologiques. Il étudie la bourgeoisie juive allemande de l'Empire et de la monarchie autrichienne : l'antisémitisme pousse les enfants des banquiers et des industriels juifs à trouver refuge dans des métiers intellectuels, littéraires, artistiques et scientifiques et à développer des visions critiques du capitalisme - et du judaïsme. Pour lui, le phénomène de « haine de soi juive » est ainsi un phénomène des années 1880-1900, étroitement lié à « un rejet ou une critique du système économique capitaliste » et à « l'intériorisation » d'un antisémitisme qui souligne la soif de l'argent et le matérialisme, qui seraient spécifiquement juifs : il y a eu selon Hans Dieter Hellige une « juxtaposition entre le conflit avec le père, l'antisémitisme et la critique anticapitaliste ${ }^{14}$ ».

La judéité est ainsi vécue uniquement comme un fardeau, un manquement dont il faudrait se débarrasser pour ne plus être coupable d'en porter les traces. L'historien Alex Bein souligne ainsi avec beaucoup de finesse que les Juifs allemands tourmentés « trouvaient justifiée la haine du Juif dans la société et la reportaient sur eux-mêmes avec un sentiment de culpabilité qu'ils expiaient en se retournant contre d'autres Juifs ou contre eux-mêmes ${ }^{15}$ ». C'est ainsi que Jacques Le Rider parle avec grande justesse de « désubstantialisation de l'identité du Juif assimilé ${ }^{16} »$.

entfliehen »), p. 216 (« sein Erbe verschmähen und seine Identität verleugnen »).

10. Lewin, Die Lösung sozialer Konflikte, p. 259.

11. Jacob Toury, "Jüdische Parteigänger des Antisemitismus », Bulletin des Leo Baeck Instituts 13, 1961, p. 323-335, ici p. 323.

12. Hans Dieter Hellige, "Generationskonflikt, Selbsthaß und die Entstehung antikapitalistischer Positionen im Judentum - Der Einfluß des Antisemitismus auf das Sozialverhalten jüdischer Kaufmanns- und Unternehmersöhne im Deutschen Kaiserreich und in der K.u.K.-Monarchie », Geschichte und Gesellschaft 5, 1979, p. 476-518, ici p. 478.

13. Voir Walter Grab, Der deutsche Weg der Judenemanzipation. 1789-1938, München, Piper, 1991, p. 179.

14. Hellige, «Generationskonflikt », p. 479, 480 et 484.

15. Alex Bein, Die Judenfrage - Biographie eines Weltproblems, t. I, Stuttgart, Deutsche VerlagsAnstalt, 1980, p. 257 ( Sie akzeptierten gewissermaßen den Judenhass der Umwelt als berechtigt und übertrugen ihn auf sich selbst mit Schuldgefühlen, die sie durch feindselige Handlungen gegen die Juden oder gegen sich selbst abbüßen ».).

16. Jacques Le Rider, Modernité viennoise et crises de l'identité juive, Paris, PUF, 1990, p. 243. 


\section{Expressions de la « haine de soi juive»}

Voyons désormais quelques-unes des échappatoires que certains Juifs allemands ont envisagées afin d'éviter le tourment intérieur, la double interrogation sur "leur appartenance au judaïsme et leur adhésion à la culture allemande ${ }^{17}$ ". Le rejet de soi a ainsi pu s'exprimer à travers la sortie de la communauté juive et la conversion, le changement de patronyme ou l'opposition contre les Juifs de l'Est fuyant notamment les pogromes en Russie.

On pourra rappeler au préalable qu'en Allemagne, le fait de quitter ou d'entrer dans une communauté religieuse est d'abord un acte administratif lié à certaines charges fiscales. On notera en outre que l'on peut abandonner sa confession d'origine sans pour autant se convertir à une autre religion, c'est-à-dire sans forcément se faire enregistrer dans une nouvelle communauté. La conversion est perçue par la communauté d'origine comme particulièrement déshonorante : la langue allemande - comme la langue française - utilise les termes durs d'apostat («Apostat ») et de renégat («Renegat »).

À la fin des années 1920, Theodor Lessing commentait dans une lettre adressée à sa femme Ada le geste de la conversion : «La plupart des jeunes gens dont je fais la connaissance voudraient se convertir au christianisme afin de se débarrasser de leurs conflits tout à fait insolubles ${ }^{18}$ ».

On connaît la lettre désespérée de Heinrich Heine à son ami Moses Moser après sa conversion ratée, ratée car ne lui ouvrant pas davantage les portes de la magistrature :

Ich bin jetzt bei Christ und Jude verhasst. Ich bereue sehr, dass ich mich getauft habe; ich seh noch gar nicht ein, dass es mir seitdem besser gegangen sey, im Gegentheil, ich habe seitdem nichts als Unglück ${ }^{19}$.

Ce qui pour Heine avait pu représenter un « Entréebillett zur europäischen Kultur » avait échoué. Plus de cent ans plus tard, en 1935, Kurt Tucholsky confiait à son ami Arnold Zweig (dans une lettre du 15 décembre 1935) : "Je suis "sorti du judaïsme" en 1911 et je sais que cela est impossible 20 ». La question qui se pose ici serait évidemment de savoir (et donc d'étudier au cas par cas) si ce geste de la conversion qui allie assimilation et disparition, intégration et dilution - si ce

17. Daniel Azuélos, "Judéité et germanité : l'impossible symbiose ? (Lion Feuchtwanger, Arnold Zweig et Jakob Wassermann) », Pardès 5, 1987, p. 161.

18. Stadtarchiv Hannover, Theodor Lessing Nachlass (StAH-ThLN) 867, Lettre adressée à Ada Lessing, portant la date du 8 janvier 1928 ( Nahezu alle jungen Menschen, die ich kennen lerne, liebäugeln mit Übertritt zum Christentum, um ihrer ganz unlösbaren Konflikte ledig zu sein ».).

19. Heinrich Heine, Lettre à Moses Moser datée du 9 janvier 1826, reproduite dans Friedrich Hirth (éd.), Heinrich Heines Briefwechsel, t. 1, München/ Berlin, Georg Müller, 1914, p. 401.

20. Kurt Tucholsky, Politische Briefe, éd. par Fritz J. Raddatz, Reinbek bei Hamburg, Rowohlt, 1969, p. 117 ("Ich bin 1911 "aus dem Judentum ausgetreten", und ich weiß, daß man das gar nicht kann »). Kurt Tucholsky quitta la communauté juive mais attendit cinq années avant de se convertir au protestantisme (voir Erich Gottgetreu, « Zum Thema des jüdischen Selbsthasses Der Fall Tucholsky versus Tucholsky », MB Mitteilungsblatt des Irgun Olej Merkas Europa 37/38, 18 septembre 1963, p. 11-13). 
geste est inévitablement l'expression d'un reniement ou d'une haine vis-à-vis de la communauté juive ou de l'identité juive.

Concernant le changement de patronyme, la sociologue Nicole Lapierre pose que celui-ci sanctionne " effectivement la rupture avec le monde juif », ces changements correspondant avant 1933 «à un effacement de l'origine et à une mise en conformité du signe avec le soi allemand revendiqué ${ }^{21}$ ». Dans l'étude qu'il consacre notamment à l'instrumentalisation antisémite de certains noms ou prénoms, Dietz Bering montre comment des patronymes ou des prénoms étaient considérés comme juifs et objets de moqueries ou d'attaques, que ces noms soient d'origine hébraïque comme Abraham, latine comme Moritz, grecque comme Isidor ou allemande comme Goldstein ${ }^{22}$. De nombreuses plaisanteries circulaient sur ces patronymes, comme la figure du «Petit Moritz», prénom moqué, car censé cacher le prénom de Moïse ${ }^{23}$. Les noms de famille pour lesquels le changement a été le plus souvent sollicité pendant la République de Weimar étaient ceux de Cohn/Kohn, Moses, Itzig ou Levi, avec ses différentes variantes Lev/w/i/y/n ${ }^{24}$. Mais malgré la forte charge antisémite, les demandes de changement de nom sont restées sur la période 1867-1933 très minoritaires et les autorisations ont été octroyées avec une grande parcimonie ${ }^{25}$.

Autre expression du rejet de l'identité juive : le rejet par les Juifs assimilés des Ostjuden qui représentent en 1910 près de $12 \%$ et en $192515 \%$ de la population juive, soit respectivement un peu plus de 70000 et de 88000 personnes ${ }^{26}$. Je commencerai par citer un épisode remontant à l'enfance de Theodor Lessing : alors que, ignorant de ses origines, il vient d'être traité de Juif à l'école, il demande à sa mère de lui expliquer ce que cela signifie : celle-ci esquive puis, plus tard, lui montre du doigt un homme passant dans la rue, vêtu d'une longue lévite noire en s'écriant : "Voilà un Juif ${ }^{27}$ ".

L'étude très précieuse que Trude Maurer a consacrée aux Ostjuden dans l'Allemagne de la République de Weimar brosse le portrait des Juifs d'Europe de

21. Nicole Lapierre, «Changement de nom : le signe, la haine, le soi », in Benbassa, Attias, La haine de soi, p. 273-292, ici p. 273.

22. Dietz Bering, Der Name als Stigma - Antisemitismus im deutschen Alltag 1812-1933, Stuttgart, Klett, 1987.

23. Dietz Bering rapporte ainsi cette mauvaise plaisanterie qui circulait : « Ein kleiner Jude wird gefragt, wie er denn heiße. "Moritz". Die witzelnde Replik des Fragers: "Ja, schon gut, aber zuhause, da nennt Dich die Mame aber doch 'Moischele', nicht ?" » (les termes yiddish " Mame » - pour mère - et « Moischele » - diminutif pour Moïse - habituellement affectueux, sont ici tournés en dérision). Ibid., p. 243.

24. Ibid., p. 222.

25. Voir aussi Lapierre, « Changement de nom », p. 282.

26. Trude Maurer, Ostjuden in Deutschland 1918-1933, Hamburg, Hans Christians, 1986, p. 72. Voir aussi Steven E. Aschheim, Brothers and Strangers - The East European Jew in German and German Jewish Consciousness 1800-1923, Wisconsin, The University of Wisconsin Press, 1982.

27. Theodor Lessing, Einmal und nie wieder, Gütersloh, Bertelsmann, 1969 [1935], p. 112 (« Ich erkundigte mich bei meiner Mutter, was ein Jude sei. Sie lachte und gab eine ausweichende Antwort. Einmal aber zeigte sie mir auf der Straße einen Mann im Kaftan und sagte :'Da geht ein Jude'. Daraus schloß ich, daß wir keine'richtigen' seien. »). 
l'Est tel que l'opinion publique allemande d'alors se les représentait ${ }^{28}$. D'après la représentation commune de l'époque, l'Ostjude était avant tout perçu comme sale, ignorant qu'il était des règles élémentaires d'hygiène ; il ne travaillait pas, était un fainéant, un vagabond, un « Schnorrer » (mendiant), voire un malfaiteur lié notamment à des affaires de mœurs ; s'il travaillait, il se cantonnait au commerce et au trafic - c'est alors un "Schacherjude », un trafiquant juif, dont l'image contraste avec celle du "bon paysan allemand" véritablement productif. D'une manière générale, il était considéré comme ayant un niveau culturel très bas et appartenant à une " race » inférieure. Or Trude Maurer souligne que cette perception de l'Ostjude aux accents fortement antisémites était en grande partie partagée par les Juifs allemands eux-mêmes ${ }^{29}$. Le sociologue Wolfgang Schivelbusch remarque ainsi qu'au cours du XIX siècle, les Juifs assimilés, cherchant à « éviter tout ce qui pouvait rappeler de près ou de loin la caricature du "Juif du ghetto" [...], sont allés jusqu'à construire un propre contretype juif presque indiscernable de l'image donnée par la propagande antisémite ${ }^{30} »$.

\section{Exemples d'intellectuels juifs allemands et autrichiens}

Je voudrais maintenant aborder le cas particulier des intellectuels juifs allemands ou autrichiens qui ont exprimé cette haine de soi et qui, pour nombre d'entre eux, ont décrit le phénomène de rejet de soi. Ce sont, pour la période considérée, toujours un peu les mêmes noms qui reviennent dans la littérature secondaire : pour l'Allemagne, Bertold Auerbach, Paul Rée, Maximilian Harden ${ }^{31}$, Walther Rathenau ${ }^{32}$, Karl Wolfskehl, Theodor Lessing, Jakob Wassermann, Carl Sternheim, Rudolf Borchardt, Max Steiner, Walter Calé, Kurt Tucholsky. Pour Vienne, ceux de Hugo von Hofmannsthal, Karl Kraus, Egon Friedell, Otto Weininger ${ }^{33}$, Arthur Trebitsch. Pour Prague, ceux de Fritz Mauthner ${ }^{34}$, Victor Adler ou Franz Werfel.

Ces hommes ont pu ressentir et exprimer un sentiment de rejet de manière passagère, comme c'est le cas pour Theodor Lessing. Certains sont sortis de la communauté juive sans se convertir (Walther Rathenau) ou se sont convertis :

28. Voir Maurer, Ostjuden in Deutschland.

29. Ibid., p. 108 : «Depuis le siècle des Lumières, les Juifs, groupe plus faible et constamment menacé, ont adopté, selon leur degré d'assimilation, les clichés des Non-Juifs ». Trude Maurer souligne cependant que cette image pouvait être nuancée, voire contrebalancée par l'évocation de l'amabilité des Ostjuden, de leur sens de la famille, de leur amour de la patrie ou de leur intelligence.

30. Wolfgang Schivelbusch, Intellektuellendämmerung - Zur Lage der Frankfurter Intelligenz in den zwanziger Jahren, Frankfurt a. M., Suhrkamp, 1985, p. 35.

31. Sur Paul Rée, Maximilian Harden, Rudolf Borchardt, Max Steiner et Walter Calé, on pourra se reporter aux portraits dressés par Theodor Lessing dans Der jüdische Selbsthass.

32. Voir notamment Peter Loewenberg, "Antisemitismus und jüdischer Selbsthass », in Reinhard Rürup, Antisemitismus und Judentum, Göttingen, Vandehoeck und Ruprecht, 1979, p. 455-475.

33. Voir notamment Jacques Le Rider, Le cas Otto Weininger. Racines de I'antiféminisme et de I'antisémitisme, Paris, PUF, 1982.

34. Voir notamment : Gershon Weiler, «Fritz Mauthner. A Study in Jewish Self-Rejection », The Leo Baeck Institute Year Book 8 (1), 1863, p. 136-148. 
au catholicisme comme Karl Kraus (qui sortit de la communauté juive en 1899, se convertit douze ans plus tard et sortit finalement aussi de l'église catholique en $1923^{35}$ ) ; au protestantisme, comme Carl Sternheim, Kurt Tucholsky, Maximilian Harden ou Egon Friedell ${ }^{36}$. D'autres enfin se sont laissé entraîner jusqu'au suicide, comme Paul Rée, Walter Calé, Max Steiner ou Otto Weininger.

Certains de ces auteurs ont exprimé ce sentiment de haine de soi par le truchement de personnages juifs, souvent caricaturaux, parfois satiriques. Je propose d'en évoquer trois exemples particulièrement prégnants.

Ludwig Jacobowski publie au début des années 1890 Werther der Jude, roman qui met en scène un jeune Juif, Leo Wolff, dont les parents sont originaires d'Europe de l'Est et qui est désireux de s'assimiler rapidement depuis qu'il fait ses études à Berlin : il est membre de la corporation estudiantine « Guestphalia », a une liaison avec une jeune Allemande blonde. Confronté à l'antisémitisme, il s'avoue son "tourment intérieur », son " mépris de soi » et voit dans la seule régénération morale des Juifs la solution à la question juive ${ }^{37}$.

Kurt Tucholsky compose quant à lui dans la Weltbühne dans les années 1920 le personnage de Monsieur Wendriner, dont le lecteur ne perçoit que le seul monologue dans les différentes situations décrites. Représentant le Spießer juif de manière caricaturale, Wendriner nous apparaît comme un homme vulgaire, désobligeant, impoli, incapable de se tenir en public, qu'il soit à la mer $^{38}$, à un enterrement (sa femme ne l'accompagne pas, elle a rendez-vous chez le couturier $^{39}$ ), chez le coiffeur ${ }^{40}$. La famille Wendriner est une famille disloquée, désunie, sans liens profonds: Wendriner trompe sa femme pendant son absence avec une jeune blonde délurée et, pour se justifier, annonce : "Elle s'est reposée à la plage. Je me suis reposé $\mathrm{ici}^{41}$; » son fils est incapable de se tenir au restaurant, met l'établissement sens dessus-dessous et Wendriner de conclure :

Ich frage mich bloß eins: diese Unbeständigkeit, diese Fahrigkeit, diese schlechten Manieren - von wem hat der Junge das ${ }^{42}-$ ?

35. Voir Grab, Der deutsche Weg, p. 154.

36. Egon Friedell se fait baptiser en 1897. II se suicide en se défenestrant quand, cinq jours après I'«Anschluss », deux SA entrent chez lui pour l'arrêter. Ibid., p. 162.

37. Ludwig Jacobowski, Werther der Jude, Berlin/ Leipzig, Schweizer, 1898 [1891], notamment p. 12, $18,42,125,145-146,151$. Le portrait que Theodor Lessing brosse de Ludwig Jacobowski au lendemain du décès de celui-ci pourrait tout à fait figurer dans La haine de soi juive. Theodor Lessing, "Ludwig Jacobowski », Ost und West - Illustrierte Zeitschrift für modernes Judentum, août 1901 (huitième cahier), col. 561-576 (Ludwig Jacobowski est décédé le 2 décembre 1900).

38. Kurt Tucholsky, « Herr Wendriner nimmt ein Bad» (1925), in Kurt Tucholsky, Gesammelte Werke in 10 Bänden, éd. par Mary Gerold-Tucholsky et Fritz J. Raddatz, t. 4, Reinbek bei Hamburg, Rowohlt, 1975 , p. $155-156$.

39. Kurt Tucholsky, « Herr Wendriner beerdigt einen » (1925), ibid., p. 203-204 (« Meine Frau hab ich zu Hause gelassen. Sie regt sich immer so auf. Beerdigungen sind nichts für Frauen. Außerdem hatte sie heute Anprobe bei der Schneiderin »).

40. Kurt Tucholsky, « Herr Wendriner lässt sich die Haare schneiden » (1925), ibid., p. 221-223.

41. Kurt Tucholsky, « Herr Wendriner betrügt seine Frau » (1925), ibid., p. 233-235 (" Sie hat sich am Strand erholt. Ich hab mich hier erholt »).

42. Kurt Tucholsky, « Herr Wendriner erzieht seine Kinder » (1925), ibid., p. 90-91. 
Le portait ainsi brossé du bourgeois juif est satirique et sans nuance, comme l'écrivait lui-même Tucholsky :

(...) der Satiriker ist ungerecht. Er kann nicht wägen - er muss schlagen. Und verallgemeinert und malt Fratzen an die Wand und sagt einem ganzen Stand die Sünden einzelner nach, weil sie typisch sind, und übertreibt und verkleinert ${ }^{43}$.

Die Satire muss übertreiben und ist ihrem tiefsten Wesen nach ungerecht ${ }^{44}$.

Évoquant Kurt Tucholsky, Marthe Robert soulignait ainsi le danger du discours satirique, " outil pratique mais douteux " qui " dénonce et se dispense d'analyser ${ }^{45}$ ». Avec Herr Wendriner, Kurt Tucholsky dresse sans nuances les travers du Juif assimilé, Erich Gottgetreu parle à ce propos d'« auto-persiflage involontaire ${ }^{46} »$.

On trouve dans les romans de Jakob Wassermann la figure du Jüdischer Selbsthasser, ainsi le personnage de Waremme/Warschauer dans Der Fall Maurizius $^{47}$ : né en Silésie, Warschauer choisit d'abord la solution de la conversion, l'abandon de toute référence juive : il s'invente une mère chrétienne, prétend être le fruit d'un adultère avec un grand propriétaire noble de Silésie ; de son père adoptif, catholique fervent, il prend le patronyme de Waremme, qui lui ouvre les portes du monde, jusqu'au pape et à l'Empereur. Il explique que c'est justement son apparence physique qui lui a permis de jouer un rôle dans la société : « La nature m'avait avantagé, j'étais blond, d'un blond authentiquement germanique, la forme de mon visage [...] n'est pas orientale ${ }^{48} \gg$ - et souligne dans une discussion avec le personnage central du roman, Etzel Andergast :

Können Sie sich vorstellen, daß ein Mensch sich selber über seine Geburt belügt? Komplizierte Sache. Der nicht sein wollen, der man ist, die Wurzel verleugnen, aus der man gewachsen ist, das heißt die eigene Haut wie einen geborgten Mantel tragen ${ }^{49}$.

La haine de soi ne s'exprime chez les intellectuels juifs allemands pas uniquement dans des textes de fiction. On connaît la charge terrible lancée par Walther Rathenau dans son " Höre Israel ", publié en 1897 sous le pseudonyme de W. Hartenau, qui n'hésite pas à décrire les Juifs de Berlin (et donc lui-même) comme

43. Kurt Tucholsky, «Politische Satire » (1919), in Tucholsky, Gesammelte Werke, t. 2, p. 171-173 (ici p. 172).

44. Kurt Tucholsky, « Was darf die Satire » (1919), ibid., p. $42-44$ (ici p. 43).

45. "'Une intelligence qui s'est trompée d'ennemi' - Marthe Robert analyse Kurt Tucholsky », propos recueillis par Nicole Zand, Le Monde des Livres, 12 mars 1982, p. 18.

46. Gottgetreu, «Zum Thema des jüdischen Selbsthasses », p. 12 (« Diese Monologe [...] enthalten aber auch ein gutes Stück - ungewollter - Selbstpersiflage »).

47. Sur Jakob Wassermann, on pourra lire mon article: Martine Benoit, «Le destin romanesque des Juifs allemands dans l'œuvre de Jakob Wassermann : entre quête d'identité et isolement », Germanica 22, 1998, p. 93-108, en ligne: [http://germanica.revues.org/1323], dernière consultation le 15 avril 2019.

48. Jakob Wassermann, Der Fall Maurizius, München, DTV, 1988 [1928], p. 311 (« Die Natur hatte mich begünstigt, ich war blond, unverfälscht germanenblond, mein Gesichtsschnitt [...] ist unorientalisch. »).

49. Ibid., p. 307. 
une « horde asiatique » et exprimer par là sa gêne liée à ce qui serait la manière ostentatoire et bruyante des Juifs allemands de se comporter en société :

Wer ihre Sprache vernehmen will, mag an Berliner Sonntagen mittags um zwölf durch die Thiergartenstraße gehen oder abends in den Vorraum eines Theaters blicken. Seltsame Vision! Inmitten deutschen Lebens ein abgesondert fremdartiger Menschenstamm, glänzend und auffällig staffirt, von heißblütig beweglichem Gebahren. Auf märkischem Sand eine asiatische Horde. Die gezwungene Heiterkeit dieser Menschen verräth nicht, wieviel alter, ungesättigter Haß auf ihren Schultern lastet ${ }^{50}$.

Dernier exemple d'un texte non fictionnel : la troublante et douloureuse autobiographie de Jakob Wassermann, Mein Weg als Deutscher und Jude, dans laquelle l'auteur, à l'instar de son personnage Waremme, souligne dans les toutes premières pages : "Mein Gesichtstypus bezichtigte mich nicht als Jude, mein Gehaben nicht, mein Idiom nicht. Ich hatte eine gerade Nase und war still und bescheiden ${ }^{51}$ ». Jakob Wassermann dresse ainsi de lui-même un portrait d'abord physique où l'on voit toute l'intériorisation des préjugés antisémites du Juif au nez protubérant, bruyant, mal élevé et jargonnant avec son yiddish. Et Wassermann de dresser le portrait de ces Juifs oublieux et souffrant de leurs origines :

Ich kenne, kannte viele, die vor Sehnsucht nach dem blonden und blauäugigen Menschen vergingen. Sie betteten sich ihm zu Füßen, sie schwangen Räucherfässer vor ihm, sie glaubten ihm aufs Wort, jedes Zucken seiner Lider war heroisch, und wenn er von seiner Erde sprach, wenn er sich als Arier auf die Brust schlug, stimmten sie ein hysterisches Triumphgeschrei an. Sie wollen nicht sie selbst sein; sie wollen der andere sein ${ }^{52}$.

La haine de soi ou comment disparaître pour devenir l'autre, comment se défaire de tout ce qui est perçu comme juif pour devenir plus allemand que l'Allemand chrétien lui-même.

\section{Conclusion}

Pour conclure, je voudrais revenir à Theodor Lessing et à ses tentatives toujours réitérées de résoudre le « conflit germano-juif ${ }^{53}$ » qui I'habitait. Dans I'un de ses derniers articles, « Ayez pitié du mulet » («Gnade dem Maultier »), Theodor Lessing revient sur cette dissonance, cette déchirure :

[Ich stehe] in einem Zeitpunkt, wo man auch von mir, wie von jedem verlangt, daß er sich in zwei Hälften auseinander legen oder analysieren lasse und daß er endlich klar sich entscheiden müsse, was er und seine Nachfahren denn eigentlich seien, ob Roß oder Esel, ob Jude oder Deutscher. Worauf ja doch schließlich die ehrliche Natur immer nur neu erwidern kann :"Ich bin wie ich bin: Ein Maultier! ${ }^{54 " .}$

50. Cité d'après Loewenberg, " Antisemitismus und jüdischer Selbsthass », p. 460-461.

51. Jakob Wassermann, Mein Weg als Deutscher und Jude, Berlin, S. Fischer, 1921, p. 12-13.

52. Ibid., p. 110.

53. Cf. Léon Poliakov, Histoire de l'antisémitisme, t. II, L'Âge de la science, Paris, Calmann-Lévy, 1981, p. 271.

54. Theodor Lessing, "Gnade dem Maultier » (1933) reproduit dans Hans Stern, Theodor Lessing Wortmeldungen eines Unerschrockenen - Publizistik aus drei Jahrzehnten, Leipzig, Kiepenheuer, 
Ce qui est intéressant de souligner dans cette citation, c'est que germanité et judéité y sont toutes deux mises sur le même plan; d'autre part, on peut noter que le terme d'« âne » est accolé à celui d'Allemand alors que Theodor Lessing emploie pour qualifier la composante juive de son être, le terme relevé et poétique de «Ross » que l'on pourrait rendre en français par le mot ancien de « palefroi ». Le propos se clôt sur l'image du mulet, croisement d'un âne et d'une jument, dont on rappellera (pour souligner la maladresse consciente ou non de cette image) qu'il ne peut se reproduire - et que l'on peut lire comme un aveu maladroit de la douleur du Juif allemand à la veille de l'accession d'Hitler au pouvoir. 